EPJ Web of Conferences 53, 08006 (2013)

DOI: $10.1051 /$ epjconf/20135308006

(C) Owned by the authors, published by EDP Sciences, 2013

\title{
Results from and prospects for the Auger Engineering Radio Array
}

\author{
A.M. van den Berg ${ }^{1, a}$ for the Pierre Auger Collaboration ${ }^{2, b}$ \\ ${ }^{1}$ Kernfysisch Versneller Instituut, University of Groningen, 9747AA Groningen, \\ The Netherlands \\ ${ }^{2}$ Av. San Martín Norte 304, (5613) Malargüe, Prov. de Mendoza, Argentina
}

\begin{abstract}
The Auger Engineering Radio Array (AERA) is one of the low-energy enhancements of the Pierre Auger Observatory. AERA is based on experience obtained with the LOPES and CODALEMA experiments in Europe and aims to study in the $\mathrm{MHz}$ region the details of the emission mechanism of radio signals from extensive air showers. The data from AERA will be used to assess the sensitivity of $\mathrm{MHz}$ radiation to the mass composition of cosmic rays. Because of its energy threshold at $2 \times 10^{17} \mathrm{eV}$ the dip region in the cosmic-ray flux spectrum can be studied in detail. We present first results of AERA and of its prototypes and we provide an outlook towards the future.
\end{abstract}

\section{INTRODUCTION}

A next generation of ground-based cosmic-ray observatories is required to address fundamental questions in astroparticle physics at extreme energies. These questions concern the origin of cosmic rays with an energy larger than $10^{19} \mathrm{eV}$, their acceleration mechanism, and their particle-particle interactions at energies far beyond the $\mathrm{TeV}$ scale, which take place at the acceleration sites and subsequently along their path through the cosmos until their detection at a ground-based observatory. Especially at energies beyond $10^{19} \mathrm{eV}$ we need improved statistics and precise information on energy, composition, and arrival direction. The detailed registration of the avalanche of secondary particles, known as the air shower, is an essential tool to infer these properties of the primary particle. At this moment the preferred method to study the nature of the primary particle is based on the determination of the longitudinal shower profile, for instance by using optical detectors which collect the ultra-violet photons emitted by ionized molecules along the path of the shower. As this light signal is very weak, the current uptime of such detection systems is limited to dark nights, causing an effective uptime of about $13 \%$. Obviously, this affects the statistics as well. In principle surface-detector arrays have an uptime of $100 \%$ and are excellent to determine both the particle flux and the arrival direction. Recently, progress has been made to enhance the sensitivity of sparse surface-detector arrays for the determination of the mass composition of ultra-high-energy cosmic rays [1].

The observation of air showers with radio-detection techniques can be done at all times. Moreover, radio signals are sensitive to the development of the electromagnetic component of particle showers in the atmosphere of the Earth. Radio detection of air showers started in the 1960's and the achievements in those days have been presented in excellent reviews by Allan [2] and Fegan [3]. More recent developments are based on initial studies performed by the LOPES [4] and the CODALEMA [5]

\footnotetext{
ae-mail: berg@kvi.nl

bFor the full authorlist see Appendix "Collaborations" in this volume

This is an Open Access article distributed under the terms of the Creative Commons Attribution License 2.0, which permits unrestricted use, distribution, and reproduction in any medium, provided the original work is properly cited.
} 
collaborations. In the last 10 years the radio-detection technique in the $\mathrm{MHz}$ region has been revived and the present radio-detector arrays for cosmic-ray research are equipped with low-noise and high-rate digital samplers. Simultaneously, the number of stations within these arrays has grown from less than ten to more than one thousand. The question to be addressed in the VHF band (30-300 MHz) is not whether extensive air showers emit radiation. At this moment the main question to answer is: can we use radio signals to determine the primary energy, the arrival direction, and the mass of cosmic rays with accuracies which are equal to or better than those obtained by other techniques? And if yes, can we build for an affordable price a huge surface-detector array based on the radio-detection technique?

The Pierre Auger Collaboration has started a research programme to answer both questions through a stepwise approach. As a first step the emission mechanisms need to be understood. As a second step the data obtained with radio-detection stations deployed at the Pierre Auger Observatory will be used to check their sensitivity with respect the determination of the air-shower parameters. Here we take advantage of the existing infrastructure of this observatory: its surface detector (SD), its fluorescence detector (FD) and its low-energy enhancements HEAT and AMIGA [6, 7]. In addition to this pure scientific question, hardware and software are being developed to study the required specifications and performance of solitary radio stations as a blueprint for a huge array. Within the same research programme, a rigorous effort has been and is being made to predict the emission processes using our current understanding of the development of air showers. Simultaneously, experiment and theory are being connected through software tools where end-to-end simulations and data analysis can be performed within the same software package [8]. All these activities fit into the Auger Engineering Radio Array (AERA) project, which started in 2009 and which is based on work within the Collaboration using various prototypes at the site of the Pierre Auger Observatory [9, 10]. In addition to this AERA project, an alternative detection system for $\mathrm{MHz}$ radio detection is being explored by the Collaboration. Within this EASIER project [11] the radio receivers are being integrated with the existing stations of the $\mathrm{SD}$ and are therefore mounted on the triangular grid of the SD with its baseline pitch of $1.5 \mathrm{~km}$.

\section{STUDIES OF THE EMISSION MECHANISM}

In the 1960's it was already conjectured, that the emission mechanisms for radio waves in the $\mathrm{MHz}$ regime are caused by the geomagnetic separation of charged particles and by the excess of negative charges at the front of the shower; see Ref. [2] for a review on this subject. Schematically, these two different emission mechanisms are depicted in Figure 1. The contribution to the emitted radio signal caused by the so-called charge-excess effect is not influenced by the geomagnetic field $\mathbf{B}$. For this case, the polarization of the electric field vector is radial with respect to the shower axis. Therefore, the direction of the polarization changes if the position of the observer changes with respect to the shower axis. In contrast to this, the geomagnetic emission mechanism induces a polarization of the electric field vector which is unidirectional. In this case, the polarization vector aligns with the direction $\mathbf{v} \times \mathbf{B}$ where $\mathbf{v}$ denotes the speed and direction of the shower penetrating the atmosphere. Therefore, for geomagnetic emission the direction of the polarization does not change as a function of the observer position.

Recent measurements performed with the radio-detection setups at Auger have been analyzed using the polarization of the radio signal as a tool to disentangle both emission mechanisms. In this analysis we defined an observable $R$, which is a measure for the signal strength in the direction perpendicular to the geomagnetic polarization, compared to the value of the total signal strength. A value of $R$ which is different from zero indicates that, in addition to the geomagnetic component, another emission mechanism contributes with a sizeable amount. Figure 2, taken from Ref. [12], displays the measured values of $R$ using a prototype setup for AERA versus model simulations performed with the MGMR code [13] (left panel) and with the REAS code [14] (right panel). These codes include the chargeexcess mechanism in the calculation of radio emission. Both the data and the predictions for $R$ deviate from zero and the correlation between the experimental and the simulated values is strong. The Pearson coefficients for the comparison of the data with the MGMR and REAS simulations are 0.64 and 0.46, 

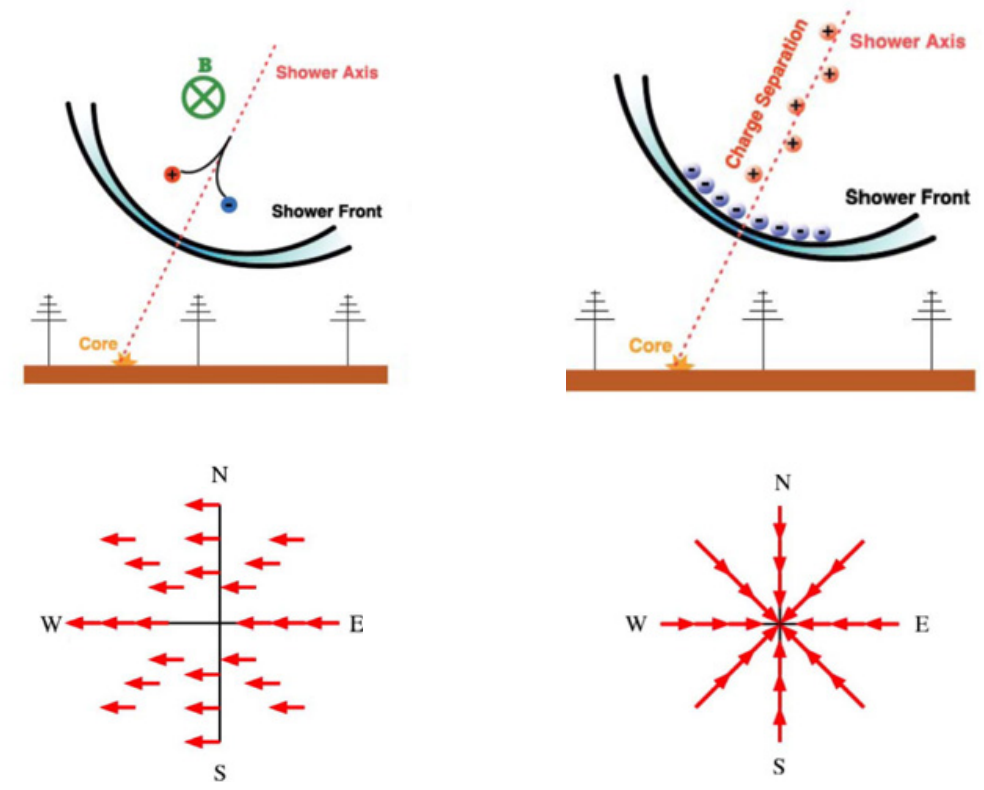

Figure 1. Schematic representation of the geomagnetic and charge-excess emission mechanisms (upper part, left and right panels, respectively). Assuming that the magnetic field $\mathbf{B}$ points north and that the shower arrives from the zenith the direction of the electric field in each of these two cases are displayed as well (lower part).
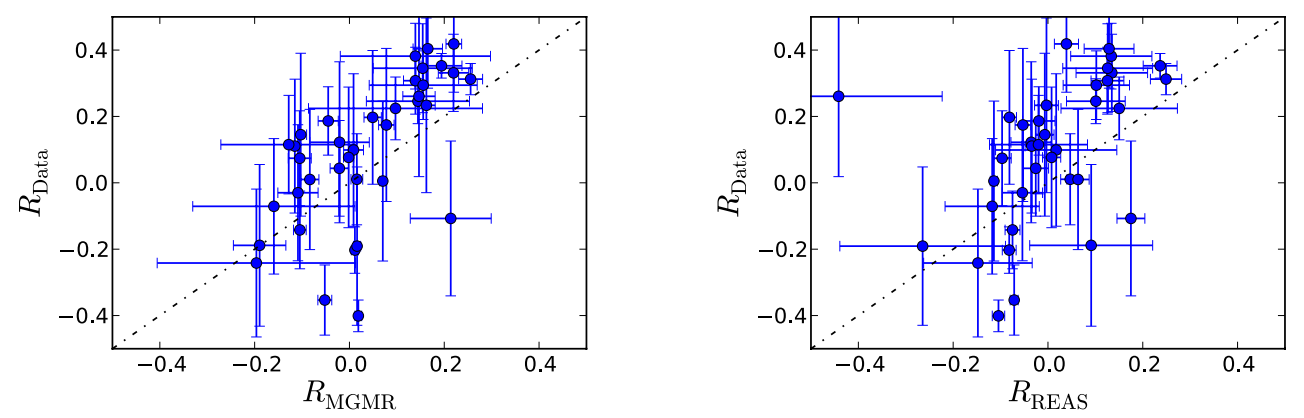

Figure 2. Measured and simulated values for the charge-excess parameter $R$; from Ref. [12].

respectively. More recent data from AERA itself which are being compared to more refined models provide further support that the charge-excess process contributes to a sizable amount [15]. Data and simulations performed by the CODALEMA collaboration [16] also show the contribution of the chargeexcess mechanism to the emission of radio waves from extensive air showers.

In addition to this charge-excess effect, the radio signals are influenced by the fact that the index of refraction of air is not equal to unity. Therefore, Cherenkov beaming effects occur. These beaming effects are expected [17] to be prominently visible at a small distance to the shower axis and at high frequencies. Depending on the composition and the energy of the primary particle, this effect strongly influences the pulse shape at a radial distance to the shower core of about 150-250 m. Measurements with the ANITA experiment have been reported [18] which show significant strength up to $1 \mathrm{GHz}$. Precision studies of air showers can be performed with LOFAR; it has a high density of radio-detection stations and it covers a wide frequency band. Recently, it has been equipped with instrumentation to 
trigger this radio telescope to register radio signals from extensive air showers [19]. Therefore, the LOFAR telescope is an excellent setup to study the shower profile close to the core of the shower.

All these results, which provide inside into the radio-emission processes, have been obtained in the last few years. Here we emphasize that several experiments independently from each other observe the same features. Moreover, it should be stressed that substantial progress has been made in the understanding of these emission mechanisms using rather different simulation codes [17]. With these tools it is now possible to make realistic simulations for a huge array of radio-detector stations.

\section{GOING TO EXTREME ENERGIES}

Another question that needs to be addressed is whether at energies beyond $10^{17} \mathrm{eV}$ the radio signal can be used to determine the energy of the primary cosmic ray to an accuracy of better than $20 \%$. Until today, this question has not been answered. In this case also, progress has been made using the setups of CODALEMA, of LOPES, and of AERA and its prototypes. However, because these setups are still rather small in size one is presently hampered by limited statistics beyond $10^{18} \mathrm{eV}$. Only preliminary data are available at this moment. The LOPES collaboration [20] has investigated the correlation of the signal strength in radio (corrected for the geomagnetic angle) versus the energy determined by the KASCADE setup. This analysis shows that up to an estimated energy of $10^{18} \mathrm{eV}$ there is a correlation. Similar results have been obtained in the framework of the CODALEMA setup; also in this analysis a correlation between signal strength in radio and energy determined with surface detectors has been found [21]. Within the AERA project an energy estimator has been defined which is based on the geomagnetic emission process. Assuming pure geomagnetic emission, the measured electric field strength is proportional to the energy of the primary particle multiplied with the sine of the geomagnetic angle. Because AERA is embedded in the AMIGA SD infill of the Pierre Auger Observatory, a careful investigation of the correlation between the electric field strength and the reconstructed energy from the surface-detector stations is possible. The first data have been obtained and are presently being analyzed.

\section{COMPOSITION STUDIES}

The emission of radio signals from air showers is governed by the development of the density distributions of electrons and positrons along the path of the shower. While fluorescence detection mainly probes the shower maximum of this distribution, radio has its largest sensitivity in the rising and falling parts of this distribution. Therefore, radio and fluorescence provide complementary information [17]. Using radio data, several techniques are being investigated to determine the shower maximum: $X_{\max }$. The first technique is a measurement of the lateral distribution function, thus the strength of the signal as a function of the distance to the shower axis. In addition to this, we can measure the pulse shape of the recorded signal. Results from the first method have been published by the LOPES collaboration [22]. The results from such an analysis and from realistic simulations can be used to optimize the bandwidth of the receivers; e.g., a bandwidth which is larger than the $30-80 \mathrm{MHz}$ presently used, or by adding another band to our receivers. Another method to study the mass composition of the primary ray is to measure the shape of the shower front at radio frequencies. Such a measurement requires precise knowledge of the arrival times of the pulses at several radio-detection stations with a resolution of a few ns and a precise determination of the shower geometry, something which is also required if we want disentangle the various emission mechanisms. A study of the shape of the wavefront has been performed by the LOPES collaboration [23]. The reported uncertainty on $X_{\max }$ by the LOPES collaboration is large; more than $100 \mathrm{~g} / \mathrm{cm}^{2}$. However, the LOPES data are strongly hampered by the noise levels at the site of LOPES. Therefore, these reported uncertainties should not be used as the ultimate achievable resolution in $X_{\max }$ that we will get with radio detection. Instead, based on simulations a value of better than $30 \mathrm{~g} / \mathrm{cm}^{2}$ is expected [24]. This expectation is close to the estimated resolution for $X_{\max }$ obtained with the FD of the Pierre Auger Observatory which is $20 \mathrm{~g} / \mathrm{cm}^{2}$ [25]. Therefore, we foresee that a 


\section{UHECR 2012}

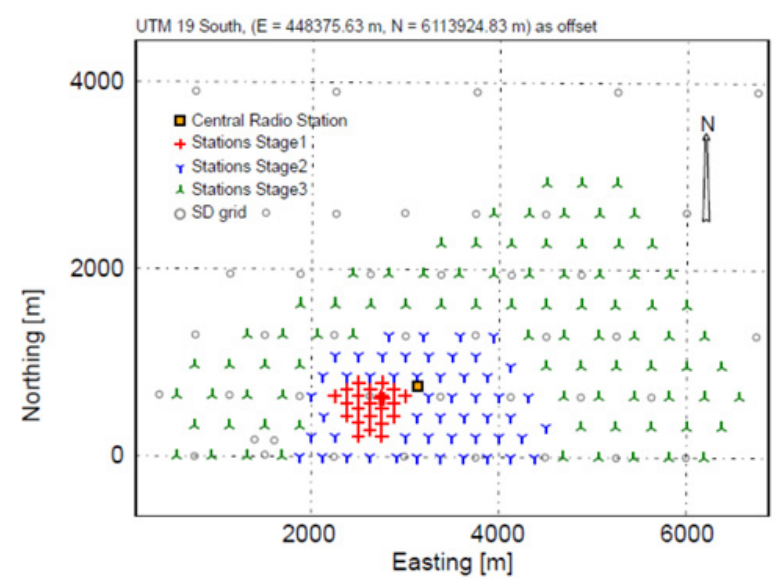

Figure 3. The layout of AERA, the core indicated by the red crosses, consists of 24 stations. The remaining stations are distributed over grids with two different values for the pitch size: $250 \mathrm{~m}$ (blue markers) and $375 \mathrm{~m}$ (green markers). The coordinates are relative to the nearest building hosting a telescope of the FD.

combination of these different techniques under development for radio detection will be needed to reach a resolution which is similar to that of the FD.

\section{PROSPECTS FOR AERA AND BEYOND}

Presently, AERA consists of 24 radio-detection stations distributed over an area of $0.5 \mathrm{~km}^{2}$. These stations are located on a triangular grid with a mutual spacing of $150 \mathrm{~m}$. In 2013 the remaining 137 stations will be installed using two different grids each with its own pitch size. Around the existing core a sector with 52 additional stations will be located having mutual separations of $250 \mathrm{~m}$. Beyond this sector, the remaining stations will have mutual separations of $375 \mathrm{~m}$; see Figure 3 . With a collecting area of about $20 \mathrm{~km}^{2}$ and a detection threshold at $10^{17} \mathrm{eV}$, this fully instrumented area allows us to collect about equal amounts of data in the energy ranges below and above $10^{18} \mathrm{eV}$. Around $10^{17.5} \mathrm{eV}$ the number of collected events per year will be more than 1,000. Very importantly, these events will be correlated with the data from the other detection systems of the Pierre Auger Observatory: SD, FD, AMIGA, and HEAT. This total data set will be used to study the sensitivity of the energy determination and the accuracy for the mass composition over the energy range from $10^{17}$ up to $10^{18.5} \mathrm{eV}$ using data from radio sensors operating in the VHF band. This energy range covers the dip region in the cosmic-ray flux spectrum. Therefore, the composition studies will provide a better insight on the transition from a galactic to an extra-galactic origin of cosmic rays in this energy region.

A technical challenge will be to test a wireless communication system which can handle the data rate from radio-detection stations, which can be as high as $1 \mathrm{kbs}^{-1}$ per station. For AERA, the wireless link needs to be stable up to a distance of $7 \mathrm{~km}$. These days, commercial off-the-shelve products seem capable of handling such a rate in a point-to-multipoint network topology. Alternatively, more advanced in-station or multi-station trigger algorithms are being developed and tested to reduce the load on the wireless network.

The physics results and the technological developments will lead to a blueprint for a radio-detection array which has a dimension of $300 \mathrm{~km}^{2}$ [26]. Subsequently, such an array will be used as a stepping stone for a huge array, focussing on the detection and physics of cosmic rays which have an energy beyond $10^{19} \mathrm{eV}$. 


\section{EPJ Web of Conferences}

\section{SUMMARY AND CONCLUSION}

In the last ten years substantial progress has been made in the understanding of radio emission from extensive air showers. In addition to the well-established geomagnetic emission process, other emission mechanisms have to be taken into account as well. Data from AERA and from other experimental setups as CODALEMA [16] and LOPES [20] will be crucial in the further refinement of the models describing radio emission in the VHF band. At present, several models are available to make detailed simulations for a huge array which will guide us to select those observables which provide us with precise information on the energy and on the composition of cosmic rays.

For the next few years it will be essential to use data from sparse arrays such as AERA and EASIER [11] side-by-side with those from dense arrays as LOFAR [19]. The sparse arrays will give information on the development of the shower profile as a function of the primary energy; the dense arrays can be used to study in detail the shape and thickness of the shower front. Both data sets will be crucial to see how radio data can be used as a tool to study particle-particle interactions at energies beyond the $\mathrm{TeV}$ regime. With the sparse and the dense arrays and the continued efforts to refine the emission and interaction models, the present momentum in radio detection will be maintained, leading to a blueprint for an array with a dimension of more than $20,000 \mathrm{~km}^{2}$.

\section{References}

[1] H. Wahlberg for the Pierre Auger Collaboration, Proc. of Science, EPS-HEP2011 052

[2] H.R. Allan, Progress in Particle and Nuclear Physics: Cosmic ray physics 10 (1971) 169

[3] D.J. Fegan, Nucl. Instr. and Methods in Phys. Res. A 662 (2012) S12

[4] H. Falcke et al., Nature 435 (2005) 313

[5] D. Ardouin et al., Astrop. Phys. 26 (2006) 341

[6] T. Hermann-Josef Mathes for the Pierre Auger Collaboration, Proc. 32nd ICRC (2011) 3, 149, arXiv:1107.4807 [astro-ph.IM]

[7] F. Sánchez for the Pierre Auger Collaboration, Proc. 32nd ICRC (2011) 3, 145, arXiv:1107.4807 [astro-ph.IM]

[8] The Pierre Auger Collaboration, Nucl. Instr. and Meth. Phys. Res. A 635 (2011) 92

[9] B. Revenu for the Pierre Auger Collaboration, Proc. 32nd ICRC (2011) 3, 172, arXiv:1107.4807 [astro-ph.IM]

[10] J.L. Kelley for the Pierre Auger Collaboration, Proc. 32nd ICRC (2011) 3, 112, arXiv:1107.4807 [astro-ph.IM]

[11] P.S. Allison for the Pierre Auger Collaboration, Proc. 32nd ICRC (2011) 3, 137, arXiv:1107.4807 [astro-ph.IM]

[12] H. Schoorlemmer for the Pierre Auger Collaboration, Nucl. Instr. and Methods in Phys. Res. A $\mathbf{6 6 2}(2012) \mathrm{S} 134$

[13] K.D. de Vries et al., Astrop. Phys. 34 (2010) 267

[14] T. Huege, R. Ulrich, R. Engel, Astropart. Phys. 30 (2008) 96

[15] The Pierre Auger Collaboration, to be published

[16] V. Marin for the CODALEMA Collaboration, Proc. 32nd ICRC (2011) 1, 291, arXiv:1109.3579 [astro-ph.IM]

[17] O. Scholten, this conference, and references cited therein

[18] S. Hoover et al., Phys. Rev. Lett. 105 (2010) 151101

[19] A. Corstanje et al., Proc. 32nd ICRC (2011) 3, 288, arXiv:1109.5805 [astro-ph.HE]

[20] The LOPES Collaboration, Astrop. Phys. 32 (2010) 294

[21] R. Dallier, 490th WE Heraeus Seminar Radio detection in astroparticle physics, Bad Honnef, October 4-6, 2011 


\section{UHECR 2012}

[22] N. Palmieri et al., Proc. 32nd ICRC (2011) 3, 60

[23] F. Schröder et al., Proc. 32nd ICRC (2011) 3, 64

[24] The LOPES Collaboration, Phys. Rev. D 85 (2012) 071101

[25] P. Facal San Luis for the Pierre Auger Collaboration, Proc. 32nd ICRC (2011) 2, 105, arXiv: 1107.4804 [astro-ph:HE]

[26] A. Haungs, this conference 